\title{
Resting-state functional magnetic resonance imaging for surgical planning in pediatric patients: a preliminary experience
}

\author{
*Jarod L. Roland, MD, ${ }^{1}$ Natalie Griffin, BA, ${ }^{2}$ Carl D. Hacker, MD, PhD, ${ }^{1}$ Ananth K. Vellimana, MD, ${ }^{1}$ \\ S. Hassan Akbari, MD, ${ }^{1}$ Joshua S. Shimony, MD, PhD, ${ }^{3}$ Matthew D. Smyth, MD, ${ }^{1,4}$ \\ Eric C. Leuthardt, MD, ${ }^{1,5-9}$ and David D. Limbrick Jr., MD, PhD ${ }^{1,4}$ \\ Departments of ${ }^{1}$ Neurological Surgery, ${ }^{3}$ Radiology, ${ }^{4}$ Pediatrics, ${ }^{5}$ Neuroscience, ${ }^{6}$ Biomedical Engineering, and ${ }^{7}$ Mechanical \\ Engineering and Materials Science; ${ }^{2}$ School of Medicine; ${ }^{8} \mathrm{Center}$ for Innovation in Neuroscience and Technology; and ${ }^{9} \mathrm{Brain}$ \\ Laser Center, Washington University in St. Louis, Missouri
}

OBJECTIVE Cerebral mapping for surgical planning and operative guidance is a challenging task in neurosurgery. Pediatric patients are often poor candidates for many modern mapping techniques because of inability to cooperate due to their immature age, cognitive deficits, or other factors. Resting-state functional MRI (rs-fMRI) is uniquely suited to benefit pediatric patients because it is inherently noninvasive and does not require task performance or significant cooperation. Recent advances in the field have made mapping cerebral networks possible on an individual basis for use in clinical decision making. The authors present their initial experience translating rs-fMRI into clinical practice for surgical planning in pediatric patients.

METHODS The authors retrospectively reviewed cases in which the rs-fMRI analysis technique was used prior to craniotomy in pediatric patients undergoing surgery in their institution. Resting-state analysis was performed using a previously trained machine-learning algorithm for identification of resting-state networks on an individual basis. Network maps were uploaded to the clinical imaging and surgical navigation systems. Patient demographic and clinical characteristics, including need for sedation during imaging and use of task-based fMRI, were also recorded.

RESULTS Twenty patients underwent rs-fMRI prior to craniotomy between December 2013 and June 2016. Their ages ranged from 1.9 to 18.4 years, and 12 were male. Five of the 20 patients also underwent task-based fMRI and one underwent awake craniotomy. Six patients required sedation to tolerate MRI acquisition, including resting-state sequences. Exemplar cases are presented including anatomical and resting-state functional imaging.

CONCLUSIONS Resting-state fMRI is a rapidly advancing field of study allowing for whole brain analysis by a noninvasive modality. It is applicable to a wide range of patients and effective even under general anesthesia. The nature of resting-state analysis precludes any need for task cooperation. These features make rs-fMRI an ideal technology for cerebral mapping in pediatric neurosurgical patients. This review of the use of rs-fMRI mapping in an initial pediatric case series demonstrates the feasibility of utilizing this technique in pediatric neurosurgical patients. The preliminary experience presented here is a first step in translating this technique to a broader clinical practice.

https://thejns.org/doi/abs/10.3171/2017.6.PEDS1711

KEY WORDS pediatric; brain mapping; resting state; functional MRI; multilayer perceptron; surgical technique

$\mathrm{F}$ UNCTIONAL mapping in neurosurgery primarily concerns localizing cerebral anatomy necessary and sufficient to perform a cognitive or motor function. This is classically achieved via invasive direct cortical stimulation (DCS), which remains the clinical gold stan- dard. Stimulation mapping in pediatric patients is technically challenging due to the dependence on adequate patient participation. Less invasive adjuncts may be useful to guide DCS or as a fallback in cases where DCS fails.

One example of a less invasive mapping procedure

ABBREVIATIONS AED = antiepileptic drug; BOLD = blood-oxygen level dependent; DCS = direct cortical stimulation; DTI = diffusion tensor imaging; ECoG = electrocorticography; $\mathrm{fMRI}$ = functional MRI; MLP = multilayer perceptron; PACS = picture archiving and communication system; rs-fMRI = resting-state fMRI; rs-MRI = resting-state MRI; RSN = resting-state network; SEEG = stereoelectroencephalography; SMA = supplementary motor area; SMN = sensorimotor network; SSEP = somatosensory evoked potential; t-fMRI = task-based fMRI.

SUBMITTED January 11, 2017. ACCEPTED June 27, 2017.

INCLUDE WHEN CITING Published online September 29, 2017; DOI: 10.3171/2017.6.PEDS1711.

* Drs. Leuthardt and Limbrick contributed equally to this work. 
involves passive recording of cortical surface potentials during task performance. Passive electrocorticography $(\mathrm{ECoG})-$ based techniques record cortical surface potentials to measure evoked activity., 2,14,16,18,22,24,33 In the setting of staged epilepsy surgery with implanted ECoG monitoring, mapping is done at the bedside, with the advantages of patient comfort, elective timing, and repetition, to achieve a high degree of accuracy. A relatively large surface area can be interrogated in parallel as opposed to the serial focal stimulation required for DCS mapping. Despite these benefits, ECoG is inherently invasive and clinically demanding, particularly in pediatric cases in which compliance issues carry the potential for significant complications involving the use of implanted electrodes. ${ }^{12}$ The use of stereoelectroencephalography (SEEG) is a growing trend, involving less invasive staged surgery for stereotactic electrode placement. For this modality also, preoperative resting-state MRI (rs-MRI) may be beneficial in helping to plan stereotactic trajectories. In the absence of staged surgery, these methods can be similarly applied during awake craniotomy with real-time results.,22,30 However, this technique reintroduces the technical difficulty of awake surgery, which is uniquely challenging in pediatric patients.

Functional MRI (fMRI) is an attractive alternative that is noninvasive, studies the entire brain simultaneously, and can be performed preoperatively. ${ }^{21}$ Task-based fMRI (t-fMRI) combines standard anatomical imaging with advanced sequences sensitive to the blood-oxygen level dependent (BOLD) signal, which is an indirect measure of neuronal function..$^{13}$ Traditional t-fMRI is performed by measuring the BOLD signal while a patient alternates between epochs of task performance and rest. Interleaved epochs of rest are used to model background signal from which the evoked task response is referenced. ${ }^{3,13}$ Common tasks include hand or foot movement for motor localization and speaking tasks to localize language. While noninvasive, $\mathrm{t}$-fMRI still depends on patient performance. ${ }^{4,9}$ Pediatric patients are often unable to adhere to a blockdesign task performance due to age, development, or other factors. In addition, pediatric patients often require sedation to tolerate MRI without excessive movement, thereby obviating any potential for cooperation with task-based methods.

Biswal and colleagues were the first to report a previously unknown relationship in the BOLD signal observed during rest epochs of t-fMRI, which led to the study of resting-state functional MRI (rs-fMRI). ${ }^{1}$ Their initial report was of temporal correlations in the BOLD signal measured in bilateral motor cortex. Further advances now allow for reliable rs-fMRI mapping to be used for clinical purposes. ${ }^{26,34,35}$ For example, the intrinsic signals measured from primary motor, primary sensory, and supplementary motor areas maintain temporally correlated BOLD activity. These areas make up the sensorimotor network (SMN), a canonical resting-state network (RSN), which is of particular interest to a neurosurgeon when planning a cranial procedure. This technique has the potential to resolve previous barriers to functional mapping in pediatric patients by performing noninvasive, task-free, and sedated functional mapping.
Here we review our use of rs-fMRI in a wide range of pediatric patients for the purpose of presurgical mapping. One previous study reported on the reliability of rs-fMRI in normal children. ${ }^{31}$ Currently there are very few reports of rs-fMRI in pediatric neurosurgery. Two prior case studies used rs-fMRI before and after corpus callosotomy; Johnston et al. found functional disconnection of RSNs between hemispheres after complete callosotomy, ${ }^{11}$ and $\mathrm{Pi}$ zoli et al. reported recovery of RSNs after successful treatment of epileptic encephalopathy by partial callosotomy. ${ }^{20}$ Vadivelu and colleagues recently reviewed the applicability of rs-fMRI in pediatric neurosurgery and presented 2 case studies. ${ }^{32}$ We do not believe that rs-fMRI, or any other method to date, has provided sufficient accuracy to completely supplant direct stimulation mapping in cases where precise localization of function is necessary for tailored cortical resection. Yet, it does appear to provide a beneficial adjunct to help guide presurgical decision making. ${ }^{15,23}$

\section{Methods}

This research was approved by the institutional review board at Washington University in St. Louis. Patients were identified via clinical chart review. We reviewed all cases involving pediatric patients undergoing craniotomy at St. Louis Children's Hospital or Barnes Jewish Hospital between December 2013 and June 2016. Only cases in which rs-fMRI was successfully processed by the clinically implemented workflow pipeline as part of the preoperative evaluation were included for further analysis. Patients 19 years or older were excluded. The decision to obtain preoperative mapping was made by the treating neurosurgeon. All surgeries were performed by one or more of the authors (D.D.L., M.D.S., and E.C.L.).

Imaging was acquired on a Siemens 3T Trio MRI scanner. Standard anatomical imaging included T1-weighted magnetization-prepared rapid acquisition gradient echo (MPRAGE) in $1 \times 1 \times 1-\mathrm{mm}$ voxels. Resting-state sequences were acquired with an echo-planar imaging (EPI) sequence sensitive to BOLD signal $(3 \times 3 \times 3-\mathrm{mm}$ voxels, TR 2.0 seconds, TE $27 \mathrm{msec}$, field of view $256 \mathrm{~mm}$, flip angle $90^{\circ}$ ). Use of sedation was determined according to clinical criteria, such as age, maturity, or any other factor assessed by the treating neurosurgeon to determine inability to tolerate an awake MRI. Choice of anesthetic was determined per clinical indication by the treating anesthesiologist.

Resting-state preprocessing was performed per previously described methodology. ${ }^{27}$ Briefly, this included compensation for slice-dependent time shifts, correction of systematic odd-even slice intensities due to interleaved acquisition, rigid body registration to correct head movement within and between sequences, spatial smoothing using a Gaussian kernel at 6-mm full width at half maximum (FWHM). We performed voxel-wise nuisance-signal regression by removing signals related to cerebrospinal fluid, white matter, and the global signal, as well as 6 movement-related parameters and their derivatives. The signal was low-pass filtered at $0.1 \mathrm{~Hz}$.

The method implemented in this clinical pipeline has been previously described in detail ${ }^{10}$ Briefly, the method uses an artificial neural network implementation known 
as the multilayer perceptron (MLP) to classify 7 canonical RSNs in each individual. The MLP is a generalization of the perceptron, which is a classical machine-learning algorithm. It is an artificial neural network that, in its most simple form, performs as a supervised binary classifier. The perceptron can generalize to multiple outputs, and it is used in this case to classify several canonical RSNs. These networks include the dorsal attention network (DAN), ${ }^{5}$ ventral attention network (VAN), ${ }^{5}$ sensorimotor network $(\mathrm{SMN}),{ }^{17}$ visual network (VIS), ${ }^{28}$ frontoparietal control network (FPC), ${ }^{6}$ language network (LAN), ${ }^{25}$ and default mode network (DMN). ${ }^{7}$ The MLP was previously trained from a large collection of functional MRI data using seeds obtained from meta-analysis. These seeds were picked to best represent the RSNs of interest. Once trained, the MLP no longer depends on seed selection and can be applied to new data to estimate network membership for each voxel of an individual's rs-fMRI. Functional maps are then overlaid on anatomical imaging for visualization. These maps are uploaded to the picture archiving and communication system (PACS) and integrated into the stereotactic neuronavigation system for intraoperative utilization.

The complete analysis has been implemented in a clinical workflow pipeline that can simply be ordered by the surgeon through the electronic medical record at our institution. The pipeline begins with image acquisition, as described above, and transfer of data to the PACS. A neuroinformatics system interfaces with the PACS to obtain and process the images (for further details see Gurney et al. ${ }^{8}$ ). The processing pipeline performs alignment of anatomical and functional images, standard resting state preprocessing, and MLP analysis. The output provides quality control metrics that are reviewed by a neuroradiologist prior to be being uploaded to the PACS for review by the surgeon and integration with the neuronavigation system. The BOLD sequences require 12 minutes of scanner time. The analysis pipeline takes less than an hour to complete. The MLP analysis does not require prior known relevant regions of interest or an expert evaluator to select the RSN of interest, as is required in more common rs-fMRI methods of seed-based or independent component analysis, respectively. Therefore, the pipeline is semiautomated, aside from evaluating quality control data, and well suited for preoperative clinical work-up.

\section{Results}

Chart review identified 156 cases in which patients underwent craniotomies during the study period. In 20 of these cases, preoperative imaging included the rs-fMRI clinical mapping pipeline, and these cases were included in the study (Table 1). The ages of the 20 patients ranged from 2 to 18 years; 12 were male and 8 were female. Sedation was used in 6 cases for which propofol was the anesthetic agent. Younger patients tended to require sedation more often, and no patient 13 years or older required sedation. Surgical indications included various tumor types, a cavernoma, and resection of seizure focus. Additional advanced imaging included diffusion tensor imaging (DTI) for tractography in 19 cases and t-fMRI in 5 cases. These demographic results are summarized in Table 1. One pa- tient underwent awake craniotomy for intraoperative stimulation mapping.

Pathologies prompting resection were most often neoplastic (11 cases), followed by epilepsy (6 cases) and vascular pathologies (3 cases). Cases in which treatment was primarily directed at seizure reduction in the setting of prior cortical resections were defined as epilepsy. Many of the patients in this series underwent additional mapping procedures. Task-based fMRI was used in 5 cases (25\%), one of which also included awake DCS mapping.

Four illustrative cases are presented below. For these cases, we selected examples demonstrating the utility of rs-fMRI in multimodal preoperative planning, guidance of cerebral mapping, and intraoperative neuronavigation for tailored cortical resections. We also picked cases that demonstrate the extremes of age for which rs-fMRI may be applied, such as at 3 years old, where other mapping modalities are either not possible or may be unreliable.

\section{Illustrative Cases}

\section{Case 1: Cavernoma Causing Epilepsy}

This 16-year-old female patient presented with a 2.5year history of medically refractory epilepsy. Her seizure semiology was of simple partial seizures. Her presenting seizures were limited to her right face and subsequently progressed to involving her right arm and leg. On physical examination she was found to be neurologically intact with no focal weakness, dysphasia, or other neurological deficits at baseline. Management with multiple antiepileptic drugs (AEDs) had failed to control her seizures, and she continued to have seizures approximately every 10 days. Diagnostic imaging revealed a lesion in the left inferior frontal lobe consistent with a cavernoma (Fig. 1A and B). The proximity of the lesion to the expected location of the hand motor cortex on the left was consistent with the presenting seizure semiology. Given the proximity of the lesion to Broca's area, we obtained functional imaging to help guide our surgical strategy.

We first obtained t-fMRI including multiple speaking and reading paradigms as well as finger tapping for speech and motor localization, respectively. Task-based fMRI was successful and localized speech to the left hemisphere and suggested a safe margin separating the cavernoma from functional cortex.

Next, rs-fMRI processing was performed, and the sensorimotor and language networks were isolated (Fig. 1C and D). The resting-state language network on the left localized to the inferior frontal lobe just anterior to the cavernoma, consistent with Broca's area, and the posterior temporal lobe, consistent with Wernicke's area. These data were consistent with the t-fMRI in localizing function and suggesting a safe margin for resection of the cavernoma. The SMN localized to the precentral and postcentral gyrus laterally, and included part of the posterior superior frontal gyrus representing the supplementary motor area (SMA). This was in congruence with t-fMRI and structural imaging data for identifying primary motor cortex.

Diffusion tensor imaging sequences were processed for tractography of the arcuate fasciculus. The arcuate fasciculus took a course just deep to the lesion in its path toward 
TABLE 1. Summary of demographic and clinical characteristics

\begin{tabular}{|c|c|c|c|c|c|c|c|c|}
\hline $\begin{array}{l}\text { Case } \\
\text { No. }\end{array}$ & $\begin{array}{c}\text { Age } \\
\text { (yrs), } \\
\text { Sex }\end{array}$ & Sedation & t-fMRI & Invasive & RSNs & Tracts & Diagnoses & Procedure \\
\hline 1 & $15.9, \mathrm{~F}$ & None & Yes & DCS & SMN, LAN & AF & Epilepsy, cavernoma & Lt frontotemporal craniotomy \\
\hline 2 & 13.1, M & None & Yes & SSEP & SMN, LAN & CST & Rt cingulate tumor & Midline frontoparietal craniotomy \\
\hline 3 & $17.6, \mathrm{~F}$ & None & No & None & SMN, LAN & none & Epilepsy, prior tumor resection & Lt frontal craniotomy \\
\hline 4 & $14.4, \mathrm{~F}$ & None & No & DCS, SSEP & SMN & CST & Epilepsy, prior tumor resection & Rt frontal craniotomy \\
\hline 5 & $6.6, \mathrm{~F}$ & Propofol & No & DCS, SSEP & SMN, LAN & CST & Epilepsy, tuberous sclerosis & Rt frontal craniotomy \\
\hline 6 & 15.7, M & None & Yes & None & SMN, LAN & CST, AF & Tumor & $\begin{array}{l}\text { Lt frontotemporoparietal crani- } \\
\text { otomy }\end{array}$ \\
\hline 7 & $8.5, \mathrm{M}$ & None & No & None & SMN, LAN & CST, AF & Tumor & Lt temporal craniotomy \\
\hline 8 & $15.0, \mathrm{M}$ & None & Yes & $\begin{array}{l}\text { ECoG, }{ }^{*} \text { DCS, } \\
\text { SSEP }\end{array}$ & SMN, LAN & CST, AF & Epilepsy, idiopathic & $\begin{array}{l}\text { Lt frontotemporoparietal crani- } \\
\text { otomy }\end{array}$ \\
\hline 9 & $7.7, \mathrm{~F}$ & None & No & None & SMN, LAN & CST, AF & Epilepsy, cavernoma & Rt frontotemporal craniotomy \\
\hline 10 & $8.5, \mathrm{~F}$ & Propofol & No & DCS, SSEP & SMN, LAN & CST, AF & Tumor & Rt frontoparietal craniotomy \\
\hline 11 & 12.2, M & None & No & None & SMN, LAN & CST, AF & Tumor & Lt parietooccipital craniotomy \\
\hline 12 & 17.1, M & None & Yes & ECoG & SMN, LAN, VIS & CST, AF, OR & Epilepsy, cavernoma & $\begin{array}{l}\text { Lt parietooccipitotemporal } \\
\text { craniotomy }\end{array}$ \\
\hline 13 & $17.9, \mathrm{M}$ & None & No & None & SMN, LAN, VIS & CST, AF, OR & Tumor & Rt temporal craniotomy \\
\hline 14 & $8.5, M$ & Propofol & No & None & SMN, LAN & CST, AF & Tumor & Rt frontotemporal craniotomy \\
\hline 15 & $9.9, \mathrm{M}$ & None & No & None & SMN, LAN & CST, AF & Tumor & Rt thalamic laser ablation \\
\hline 16 & 17.8, M & None & No & None & SMN, LAN, VIS & CST, AF, OR & Tumor & Rt occipital craniotomy \\
\hline 17 & $18.4, \mathrm{~F}$ & None & No & None & SMN, LAN & CST, AF & $\begin{array}{l}\text { Epilepsy, prior cavernoma } \\
\text { resection }\end{array}$ & Rt frontal craniotomy \\
\hline 18 & $3.4, \mathrm{M}$ & Propofol & No & None & SMN, LAN & CST & Tumor & Rt occipital craniotomy \\
\hline 19 & $12.8, \mathrm{M}$ & Propofol & No & None & SMN, LAN, VIS & CST, AF, OR & Tumor & Rt parietal needle biopsy \\
\hline 20 & $1.9, \mathrm{~F}$ & Propofol & No & $\begin{array}{l}\text { ECoG, DCS, } \\
\text { SSEP† }\end{array}$ & DMN, LAN & CST & Epilepsy, tuberous sclerosis & Lt frontoparietal craniotomy \\
\hline
\end{tabular}

$\mathrm{AF}=$ arcuate fasciculus; $\mathrm{CST}$ = corticospinal tract; $\mathrm{DMN}=$ default motor network; OR = optic radiation; LAN = language network; RSNs = resting-state networks; SMN = sensorimotor network, VIS = visual network.

* ECoG monitoring was used for epileptic focus localization, but bedside cortical stimulation monitoring was not performed in this patient.

$\dagger$ SSEPs failed to localize the central sulcus in this patient.

Broca's area. These data provided reassuring evidence for a safe resection avoiding motor and speech cortex. Given the patient's age and maturity level, we recommended an awake craniotomy for intraoperative speech mapping and complete resection of the cavernoma.

She tolerated awake surgery well without complication. We began stimulation mapping near the site identified by preoperative rs-fMRI and quickly identified eloquent cortex. Positive mapping (stimulation that disrupted function) of speech was identified at the inferior frontal lobe just anterior to the cavernoma. This corresponded well to the classical description of Broca's area. Intraoperative navigation confirmed this area to be consistent with the inferior frontal part of the language RSN identified by the MLP, as shown in Fig. $1 C$ and D.

On postoperative Day 1 the patient's speech remained fluently intact, but she was noted to have mild right facial weakness. She was treated with a tapering dose of dexamethasone and discharged home in good condition. On outpatient follow-up, her mild facial weakness completely resolved. At last follow-up, 2 years postoperatively, she remained seizure free and without neurological deficit.

\section{Case 4: Epilepsy a Decade After Tumor Resection}

This 14-year-old female patient had undergone right frontal craniotomy at 3 years of age for resection of a primitive neuroectodermal tumor. After successful completion of chemotherapy and radiation treatment, she did very well for a long period of time. At the age of 10 years, she began having seizures, which were refractory to medical management, having persisted despite treatment with multiple AEDs. Electroencephalography (EEG) localized her seizures to the right frontal lobe. Structural MRI demonstrated postsurgical changes including encephalomalacia and gliosis in the right frontal lobe (Fig. 2A and B).

Her presurgical evaluation was notable for mild motor dexterity deficits in her left upper extremity, but demonstrated full strength in power throughout. Given the degree of MRI changes extending posteriorly toward the area of expected primary motor cortex and the potential for cortical reorganization resulting from prior surgery at such a young age, we elected to obtain functional imaging of the motor system. We obtained rs-fMRI of her sensorimotor network and tractography of her corticospinal tracts. The 

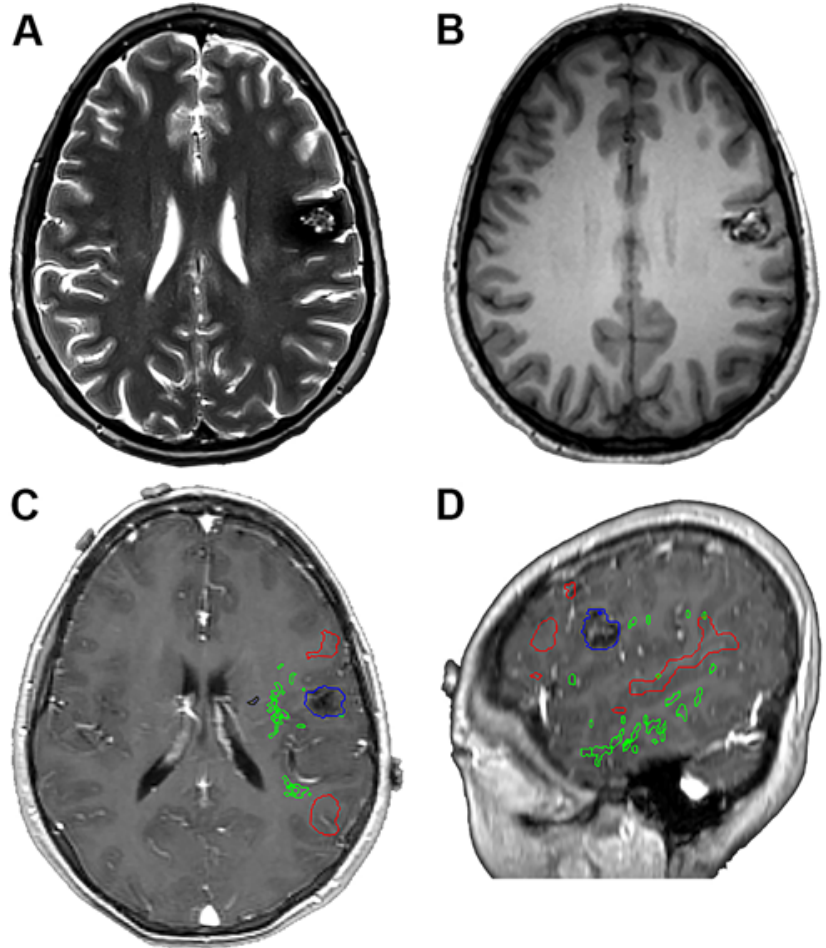

FIG. 1. Case 1. Structural MR images (A and $B$ ) and rs-fMR images (C and $D$ ) obtained in a 16 -year-old female patient. The axial T2-weighted (A) and T1-weighted (B) MR images reveal the classic popcorn-like appearance of a cavernoma; an area of signal hypointensity representing a hemosiderin ring can be seen surrounding the lesion on the T2-weighted image (A). The lesion is located in the left posterior inferior frontal gyrus and is outlined in blue in Panels $\mathrm{C}$ and $\mathrm{D}$. Language areas are identified by the language RSN outlined in red on axial (C) and sagittal (D) T1 with gadolinium contrast images, localizing Broca's area in the inferior frontal lobe just in front of the lesion and Wernicke's area in the superior temporal gyrus. Tractography of the arcuate fasciculus is outlined in green and seen passing just deep to the lesion in the axial plane. Figure is available in color online only.

SMN localized to the bilateral precentral and postcentral gyrus. There was good concordance between the corticospinal tracts terminating at cortical locations outlined by the SMN. Co-registration with structural imaging suggested a safe border for gross-total resection of the pathological frontal lobe anterior to the primary motor strip (Fig. $2 \mathrm{C}$ and $\mathrm{D})$.

We proceeded with right craniotomy for frontal lobectomy and asleep motor mapping. The patient tolerated the surgery well without complication. Surgery was performed under general anesthesia with intraoperative monitoring of somatosensory evoked potentials (SSEPs) to localize the central sulcus and cortical stimulation mapping with electromyographic monitoring to localize primary motor cortex. Intraoperative mapping localized the central sulcus by SSEPs and primary motor cortex by motor evoked potentials (MEPs) elicited from stimulation of the precentral gyrus. Neuronavigation confirmed good correspondence to the SMN identified by rs-fMRI mapping.

In the immediate postoperative period, the patient suffered a period of aphasia and right hemiparesis, consistent with an SMA syndrome. This correlated with the
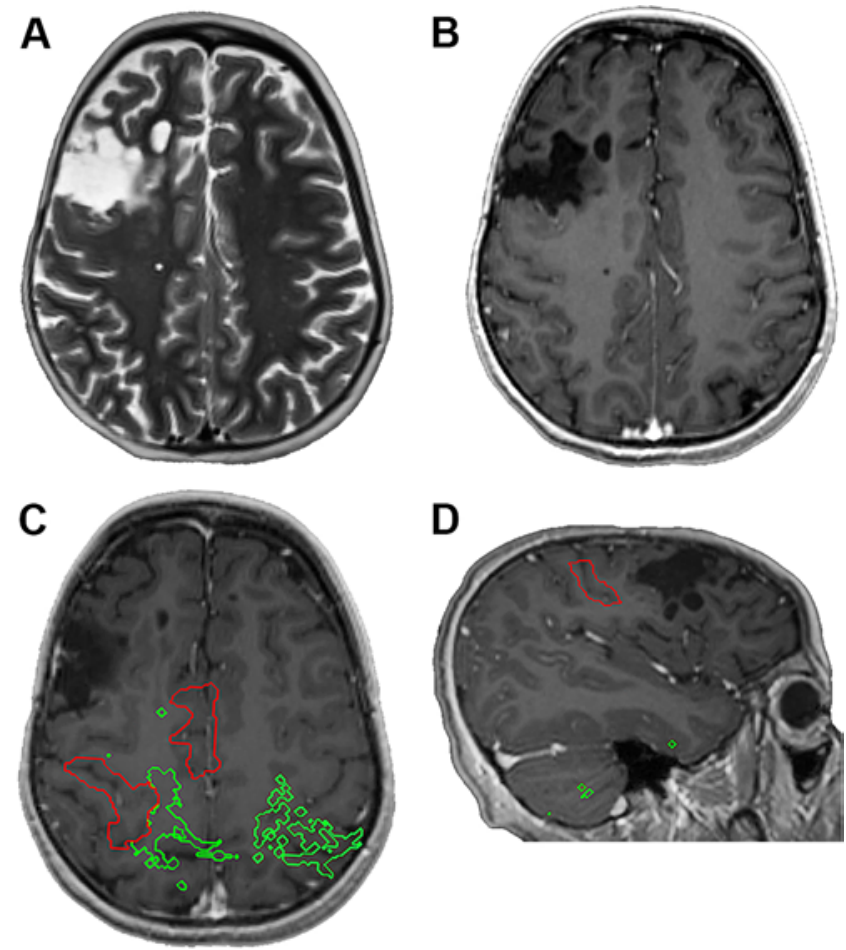

FIG. 2. Case 4. Structural MR images (A and B) and rs-fMR images (C and D) obtained in a 14-year-old female patient. The axial T2-weighted (A) and T1-weighted (B) MR images reveal encephalomalacia in the right frontal lobe consistent with previous tumor resection. The SMN is outlined in red and overlaid on axial (C) and sagittal (D) Gd-enhanced T1-weighted images identifying the pre- and postcentral gyrus and the SMA. Right and left motor tractography is also outlined in green. Figure is available in color online only.

intended frontal lobectomy including the SMA region. At the 3-month follow-up visit her speech and motor strength had completely recovered to her neurological baseline and she had full power throughout bilateral upper and lower extremities.

\section{Case 18: Mapping in a 3-Year-Old Child}

This 3-year-old boy presented with behavioral changes, irritability, and vomiting. Neuroimaging revealed a large enhancing dura-based lesion arising from the pineal region tracking along the falx cerebri, tentorium cerebelli, and basal cisterns and causing mass effect on the brainstem (Fig. 3A and B). The mass effect caused occlusion of the cerebral aqueduct resulting in obstructive hydrocephalus. The child underwent staged procedures for treatment. Initially a left frontal endoscopic approach was performed with biopsy of the mass and endoscopic third ventriculostomy for treatment of obstructive hydrocephalus. Given the size of the lesion and his young age, he returned for a right occipital craniotomy and subtotal resection. He tolerated these procedures well without complication.

Prior to the final stage of his surgery, he underwent imaging with rs-fMRI mapping of his SMN and tractography of his corticospinal tracts. The SMN localized to the bilateral precentral and postcentral gyrus and showed good concordance with corticospinal tracts terminating near 

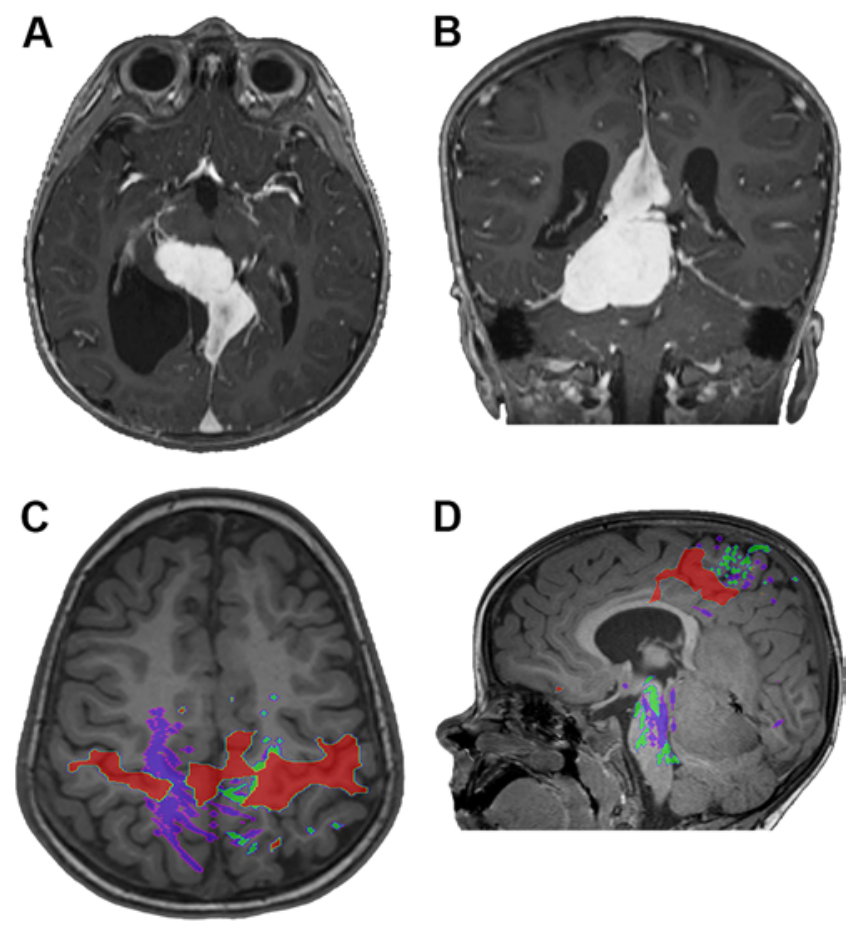

FIG. 3. Case 18. Structural MR images (A and B) and rs-fMR images ( $C$ and D) obtained in a 3-year-old male patient. The axial (A) and coronal (B) T1-weighted with gadolinium contrast MR images reveal a large dura-based legion with its epicenter in the pineal region. The SMN is overlaid in red in axial (C) and sagittal (D) images. Right and left motor tractography is also overlaid in purple and green, respectively. Figure is available in color online only.

cortex outlined by the SMN (Fig. 3C and D). His corticospinal tracts followed the severe displacement of his midbrain caused by tumor mass effect. Upon review of his imaging, a surgical approach that did not approach eloquent cortex was decided upon, and he underwent suboccipital craniotomy for final resection.

\section{Discussion}

Here we demonstrate the application of an automated rs-fMRI analysis pipeline for RSN localization in individual patients for the purpose of preoperative cerebral mapping. The advantages of resting-state paradigms for clinical mapping may be more impactful in pediatric patients than in their adult counterparts. While rs-fMRI has the potential to provide meaningful benefits in various aspects of adult and pediatric neurosurgery, we believe the mapping challenges unique to pediatric patients correspond to the inherent advantages of rs-fMRI. Specifically, pediatric patients often lack the ability to cooperate fully with strict block-paradigm task performance as required by t-fMRI or evoked electrophysiology-based mapping techniques. Patient training and experienced staff may be sufficient to overcome some degree of maturity-related limitations. However, patients at the lower age limits may present challenges even to the most experienced staff. ${ }^{28}$ Beyond the challenges of young age, cognitive deficits, which are common in pediatric neurosurgical patients, may also present challenges; in pediatric patients with cognitive deficits, sedation is often necessary to obtain structural imaging. Pediatric brain tumors are often complicated by hydrocephalus, which would additionally decrease the patient's ability to cooperate with t-fMRI.

Our results demonstrate the ability to perform RSN analysis even in the setting of general anesthesia for clinical evaluation. Our initial experience included $6(30 \%)$ patients who required sedation for preoperative imaging, which included the resting-state BOLD acquisition. All patients in this series received propofol for anesthesia. While sedation may have an impact on the magnitude of measured BOLD signal, it has been previously reported that the temporal and spatial correlation structure persists in many networks. ${ }^{19}$ Therefore, RSN localization remains easily feasible in the sedated patient.

In addition to alleviating any anxiety of the patient in the scanner, sedation also largely reduces motion. The imaging sequences required for BOLD acquisition are exquisitely sensitive to head motion. Accordingly, a significant effort in resting-state analysis is devoted to controlling for and removing effects of head motion in image preprocessing. Common techniques of motion correction were performed in our protocol, including correction of rigid body registration within and between sequences and regression of nuisance variables obtained from 6 degrees of movement parameters and their derivatives. While these procedures are still employed to help remove nonphysiologic noise from other sources in the measured signal, the artifacts from movement are largely lacking in sedated patients.

The SMN, one of several well-described RSNs, identifies primary motor and sensory regions as well as the SMA. Other RSNs are similarly identifiable, such as the language and visual networks, as mentioned briefly earlier in this paper. These networks often identify primary sensory or motor areas as well as multimodal cortical areas involved in higher-order cognitive processes. Resting state fMRI typically identifies the cortical gray matter and not the underlying white matter tracts. Therefore, as in the cases described herein, DTI-based tractography may be a complementary adjunct to be considered for certain cases. However, the relationship between lesions, mass effect, edema, and RSNs is currently unclear. For this reason, relying on RSN analysis alone in the setting of infiltrative tumors is to be performed with caution.

The functionality of rs-fMRI in patients under sedation similarly allows for application to individuals at extremely young ages. In this series, our youngest patient was 1.9 years old at the time of rs-fMRI mapping. Two patients were younger than 5 years of age (the other being 3.4 years), which is the previously reported youngest age of $\mathrm{t}$-fMRI performance. ${ }^{28}$ The 20 individuals in this series represent a relatively even distribution of ages throughout pediatric age range (1.9-18.4 years).

In the exemplar cases, rs-fMRI mapping was requested as part of the preoperative evaluation to help guide decisions for surgical strategy. In the first case (Case 1), a young girl presented with epilepsy associated with a cavernoma near suspected eloquent speech cortex. We used the preoperative rs-fMRI data to help predict our ability to achieve such a resection without introducing a deficit. 
In this case, language localized 1 gyrus anterior to the lesion (Fig. 1), suggesting that a subpial dissection up to the separating sulcus might be possible. During the awake craniotomy, we directed our stimulation mapping first to the areas identified by rs-fMRI, which was integrated in our frameless neuronavigation. We then confirmed the functional boundaries and completed the planned resection without introducing permanent deficit.

In the second case (Case 4), gliosis from a prior tumor resection performed at a young age raised concern for anatomical localization of motor cortex. Therefore, we obtained preoperative rs-fMRI to help identify primary motor cortex. In this case, the lesion extended to the gyrus immediately anterior to the primary motor region and immediately adjacent to the supplementary motor area (Fig. 2). We performed asleep motor stimulation mapping, directed by the rs-fMRI data integrated in our neuronavigation, and confirmed motor cortex in the previously identified location and not involving the anterior cortex that was affected by the lesion. Our resection did include the SMA identified by rs-fMRI, and the patient experienced a transient SMA syndrome. She recovered completely and sustained no permanent deficit.

The third case (Case 18) included resting-state mapping of the SMN in a 3-year-old child who required sedation for MRI. This is a pediatric case in which the patient would not be a candidate for an awake surgery or able to participate in any task-based mapping procedure. However, rs-fMRI mapping is feasible in such cases. DTI processing was included with rs-fMRI prior to the final stage of surgery. Tractography did overlap with the sensorimotor RSN, but also included the gyri anterior and posterior to the precentral and postcentral gyri, respectively. At the time of imaging, we had not yet committed to the surgical approach to be used. We obtained resting-state mapping at this time as only a minimal amount of additional scanner time was required and we wanted to avoid the need for an additional session of sedated imaging. After final review of this imaging, the surgical approach decided upon did not require navigating functional cortex.

This study includes the usual limitations inherent to retrospective review. To limit recall bias, we systematically manually reviewed all craniotomy procedures from an administrative database to identify all that included restingstate mapping. These data do not inform us as to the rate of failed RSN analysis, whether it be for excessive patient motion, such as in the nonsedated patient, technical errors in MRI acquisition, or methodological failures. Additionally, in this report of our early experience it is possible that cases were preferentially selected where rs-fMRI was expected to succeed or avoided where it may be expected to fail. For these reasons, care should be taken in extrapolating results to the general population. Further analysis with functional outcome measures and direct comparisons to the clinical gold standard of DCS mapping are required to better inform our interpretation of RSNs and their relationship to eloquent cortex. To compare DCS to MLP mapping, an accurate method for identifying the site of stimulation on the cortical surface that can be localized to imaging space is required. While technically feasible, such data were not available in this retrospective report.
We look forward to advancing these analyses in future endeavors.

\section{Conclusions}

The current study provides preliminary data for the basis of further research on the translation of rs-fMRI into clinical practice, specifically in pediatric neurosurgical patients. The innovation of applying a machine-learning algorithm to map cerebral networks on an individual basis and in a clinically implemented pipeline is fundamental to the translation of rs-fMRI analysis to the clinical setting. Here we have demonstrated the application of noninvasive whole brain mapping in a wide range of pediatric patients prior to neurosurgical intervention. Such results are immediately available to the treating surgeon and may serve as an adjunct in clinical decision making and consideration of surgical planning. As a clinical tool, rs-fMRI fills a gap in the armamentarium of pediatric neurosurgeons where other techniques are not applicable or are frequently unsuccessful in the very young or cognitively incapable. Restingstate fMRI may be a useful adjunct in combination with other modalities, such as t-fMRI, DTI for tractography, invasive ECoG monitoring, or awake craniotomy and cortical stimulation. When acquired alongside routine clinical imaging, rs-fMRI provides an efficient means of obtaining additional data to increase the neurosurgeon's confidence with congruent data localizing functional cortex. We do not propose rs-fMRI as a replacement for stimulation mapping, as passive mapping procedures commonly identify regions related to, but not necessarily sufficient for, cognitive function.

\section{Acknowledgments}

This work was supported by NIH R25NS090978-01 (J.L.R.) and NIH R21CA159470 (E.C.L.).

\section{References}

1. Biswal B, Yetkin FZ, Haughton VM, Hyde JS: Functional connectivity in the motor cortex of resting human brain using echo-planar MRI. Magn Reson Med 34:537-541, 1995

2. Breshears J, Sharma M, Anderson NR, Rashid S, Leuthardt EC: Electrocorticographic frequency alteration mapping of speech cortex during an awake craniotomy: case report. Stereotact Funct Neurosurg 88:11-15, 2010

3. Buchbinder BR: Functional magnetic resonance imaging, in Masdeu J, Gonzalez RG (eds): Handbook of Clinical Neurology. Neuroimaging, Part I. Amsterdam: Elsevier, 2016, Vol 135, pp 61-92

4. Byars AW, Holland SK, Strawsburg RH, Bommer W, Dunn RS, Schmithorst VJ, et al: Practical aspects of conducting large-scale functional magnetic resonance imaging studies in children. J Child Neurol 17:885-890, 2002

5. Corbetta M, Shulman GL: Control of goal-directed and stimulus-driven attention in the brain. Nat Rev Neurosci 3:201-215, 2002

6. Dosenbach NU, Fair DA, Miezin FM, Cohen AL, Wenger KK, Dosenbach RA, et al: Distinct brain networks for adaptive and stable task control in humans. Proc Natl Acad Sci U S A 104:11073-11078, 2007

7. Greicius MD, Supekar K, Menon V, Dougherty RF: Restingstate functional connectivity reflects structural connectivity in the default mode network. Cereb Cortex 19:72-78, 2009 
8. Gurney J, Olsen T, Flavin J, Ramaratnam M, Archie K, Ransford J, et al: The Washington University Central Neuroimaging Data Archive. Neuroimage 144 (Pt B):287-293, 2017

9. Håberg A, Kvistad KA, Unsgård G, Haraldseth O: Preoperative blood oxygen level-dependent functional magnetic resonance imaging in patients with primary brain tumors: clinical application and outcome. Neurosurgery 54:902-915, 2004

10. Hacker CD, Laumann TO, Szrama NP, Baldassarre A, Snyder AZ, Leuthardt EC, et al: Resting state network estimation in individual subjects. Neuroimage 82:616-633, 2013

11. Johnston JM, Vaishnavi SN, Smyth MD, Zhang D, He BJ, Zempel JM, et al: Loss of resting interhemispheric functional connectivity after complete section of the corpus callosum. J Neurosci 28:6453-6458, 2008

12. Johnston JM Jr, Mangano FT, Ojemann JG, Park TS, Trevathan E, Smyth MD: Complications of invasive subdural electrode monitoring at St. Louis Children's Hospital, 19942005. J Neurosurg 105 (5 Suppl):343-347, 2006

13. Khanna N, Altmeyer W, Zhuo J, Steven A: Functional neuroimaging: fundamental principles and clinical applications. Neuroradiol J 28:87-96, 2015

14. Korostenskaja M, Chen PC, Salinas CM, Westerveld M, Brunner P, Schalk G, et al: Real-time functional mapping: potential tool for improving language outcome in pediatric epilepsy surgery. J Neurosurg Pediatr 14:287-295, 2014

15. Lee MH, Miller-Thomas MM, Benzinger TL, Marcus DS, Hacker CD, Leuthardt EC, et al: Clinical resting-state fMRI in the preoperative setting: are we ready for prime time? Top Magn Reson Imaging 25:11-18, 2016

16. Leuthardt EC, Miller K, Anderson NR, Schalk G, Dowling $\mathrm{J}$, Miller J, et al: Electrocorticographic frequency alteration mapping: a clinical technique for mapping the motor cortex. Neurosurgery 60 (4 Suppl 2):260-271, 2007

17. Ma L, Narayana S, Robin DA, Fox PT, Xiong J: Changes occur in resting state network of motor system during 4 weeks of motor skill learning. Neuroimage 58:226-233, 2011

18. Miller KJ, Abel TJ, Hebb AO, Ojemann JG: Rapid online language mapping with electrocorticography. J Neurosurg Pediatr 7:482-490, 2011

19. Palanca BJA, Mitra A, Larson-Prior L, Snyder AZ, Avidan MS, Raichle ME: Resting-state functional magnetic resonance imaging correlates of sevoflurane-induced unconsciousness. Anesthesiology 123:346-356, 2015

20. Pizoli CE, Shah MN, Snyder AZ, Shimony JS, Limbrick DD, Raichle ME, et al: Resting-state activity in development and maintenance of normal brain function. Proc Natl Acad Sci U S A 108: 11638-11643, 2011

21. Raichle ME: A brief history of human brain mapping. Trends Neurosci 32:118-126, 2009

22. Roland J, Brunner P, Johnston J, Schalk G, Leuthardt EC: Passive real-time identification of speech and motor cortex during an awake craniotomy. Epilepsy Behav 18:123-128, 2010

23. Roland JL, Hacker CD, Breshears JD, Gaona CM, Hogan RE, Burton $\mathrm{H}$, et al: Brain mapping in a patient with congenital blindness - a case for multimodal approaches. Front Hum Neurosci 7:431, 2013

24. Schalk G, Leuthardt EC, Brunner P, Ojemann JG, Gerhardt LA, Wolpaw JR: Real-time detection of event-related brain activity. Neuroimage 43:245-249, 2008

25. Sestieri C, Corbetta M, Romani GL, Shulman GL: Episodic memory retrieval, parietal cortex, and the default mode network: functional and topographic analyses. J Neurosci 31:4407-4420, 2011
26. Shimony JS, Zhang D, Johnston JM, Fox MD, Roy A, Leuthardt EC: Resting-state spontaneous fluctuations in brain activity: a new paradigm for presurgical planning using fMRI. Acad Radiol 16:578-583, 2009

27. Shulman GL, Pope DL, Astafiev SV, McAvoy MP, Snyder AZ, Corbetta M: Right hemisphere dominance during spatial selective attention and target detection occurs outside the dorsal frontoparietal network. J Neurosci 30:3640-3651, 2010

28. Shurtleff H, Warner M, Poliakov A, Bournival B, Shaw DW, Ishak G, et al: Functional magnetic resonance imaging for presurgical evaluation of very young pediatric patients with epilepsy. J Neurosurg Pediatr 5:500-506, 2010

29. Sylvester CM, Jack AI, Corbetta M, Shulman GL: Anticipatory suppression of nonattended locations in visual cortex marks target location and predicts perception. J Neurosci 28:6549-6556, 2008

30. Taplin AM, de Pesters A, Brunner P, Hermes D, Dalfino JC, Adamo MA, et al: Intraoperative mapping of expressive language cortex using passive real-time electrocorticography. Epilepsy Behav Case Rep 5:46-51, 2016

31. Thomason ME, Dennis EL, Joshi AA, Joshi SH, Dinov ID, Chang C, et al: Resting-state fMRI can reliably map neural networks in children. Neuroimage 55:165-175, 2011

32. Vadivelu S, Wolf VL, Bollo RJ, Wilfong A, Curry DJ: Resting-state functional MRI in pediatric epilepsy surgery. Pediatr Neurosurg 49:261-273, 2013

33. Wu M, Wisneski K, Schalk G, Sharma M, Roland J, Breshears J, et al: Electrocorticographic frequency alteration mapping for extraoperative localization of speech cortex. Neurosurgery 66:E407-E409, 2010

34. Zhang D, Johnston JM, Fox MD, Leuthardt EC, Grubb RL, Chicoine MR, et al: Preoperative sensorimotor mapping in brain tumor patients using spontaneous fluctuations in neuronal activity imaged with functional magnetic resonance imaging: initial experience. Neurosurgery 65:226, 2009

35. Zhang D, Raichle ME: Disease and the brain's dark energy. Nat Rev Neurol 6:15-28, 2010

\section{Disclosures}

Dr. Leuthardt reports a patent holder relationship with Washington University. Dr. Limbrick reports support of non-study-related clinical or research effort from Medtronic, Inc.; Karl Storz, Inc.; and Microbot, Inc.

\section{Author Contributions}

Conception and design: Roland, Shimony, Smyth, Leuthardt, Limbrick. Acquisition of data: Roland, Griffin, Vellimana, Akbari, Limbrick. Analysis and interpretation of data: Roland, Griffin, Hacker, Shimony, Leuthardt, Limbrick. Drafting the article: Roland. Critically revising the article: Roland, Griffin, Hacker, Vellimana, Akbari, Shimony, Smyth, Leuthardt. Reviewed submitted version of manuscript: all authors. Approved the final version of the manuscript on behalf of all authors: Roland. Statistical analysis: Limbrick. Study supervision: Leuthardt.

\section{Correspondence}

Jarod L. Roland, Campus Box 8057, Washington University in St. Louis, 660 South Euclid Ave., St. Louis, MO 63110. email: rolandj@wustl.edu. 